\title{
Linear Ion Trap Fabricated Using Rapid Manufacturing Technology
}

\author{
Adam T. Clare, ${ }^{\mathrm{a}}$ Liang Gao, ${ }^{\mathrm{c}}$ Boris Brkić, ${ }^{\mathrm{b}}$ Paul R. Chalker, ${ }^{\mathrm{a}}$ and \\ Stephen Taylor ${ }^{\mathrm{b}}$ \\ ${ }^{a}$ Department of Engineering, University of Liverpool, Liverpool, United Kingdom \\ ${ }^{\mathrm{b}}$ Department of Electrical Engineering and Electronics, University of Liverpool, Liverpool, United Kingdom \\ ${ }^{c}$ Department of Chemistry, Purdue University, West Lafayette, Indiana, USA
}

This article presents the design, construction, and test results of a linear ion trap, fabricated using digital light processing (DLP), which is a low-cost 3D layer-based manufacturing technique. The ion trap was incorporated into a portable mass spectrometer system and experimental mass spectrum was obtained for methamphetamine ( $\mathrm{m} / \mathrm{z} 182)$, cocaine $(\mathrm{m} / \mathrm{z} 304)$, and rhodamine B $(\mathrm{m} / \mathrm{z} 443)$, with a maximum observed resolution (FWHM) of 260. For rhodamine B, tandem MS capability is also demonstrated. The mass range (and resolution at higher $\mathrm{m} / \mathrm{z}$ ) of the instrument is also demonstrated by spectrum obtained from Ultramark $(\mathrm{m} / \mathrm{z}$ 1621). The spectra obtained for the DLP trap occur at a considerably lower rf voltage than a rectilinear ion trap of similar size, which is a consequence of the hyperbolic electrode geometry and, hence, smaller $r_{0}$ in the DLP case. High mass range with low voltage operation is especially important with regard to 'in the field' applications requiring low power consumption for extended periods of operation. (J Am Soc Mass Spectrom 2010, 21, 317-322) (c) 2010 American Society for Mass Spectrometry

$\mathrm{T}$ The development of miniature mass spectrometers has advanced rapidly in recent years. A particular focus has been on building portable systems for a wide variety of 'in the field' measurements. These lines of investigation are driven by several applications, including environmental analyses and narcotics detection [1, 2]. However, these applications have similar demands to laboratory-based mass spectrometry, namely: mass discrimination, high sensitivity, and rapid operation. To meet these demands, the miniaturization of mass spectrometer components has been pursued, especially in the case of mass analyzers. Experimental miniature and microelectromechanical systems (MEMS) type quadrupole mass spectrometer (QMS) systems have been reported with quadrupole mass filters [3-5], time-offlights [6, 7], synchronous ion shield mass analyzers [8], and various models of ion traps [9-12]. However, for field applications, the use of miniature ion traps has become more widespread in recent years $[13,14]$. This is due to their larger mass range, inherent tandem MS capability, and reduced vacuum pumping requirements [15].

Rapid manufacturing (RM) or layer based manufacturing techniques (LBMTs) are a class of technologies that allow digital manufacture without the need for tooling. Instead of removing material via a reductive process, there is a repetitive addition of material in a layer-wise manner. This permits design freedoms, which

Address reprint requests to Dr. S. Taylor, Department of Electrical Engineering and Electronics, University of Liverpool, Brownlow Hill, Liverpool L69 3GJ, United Kingdom. E-mail: s.taylor@liv.ac.uk are not achievable by conventional means. Components manufactured in this way are directly manufactured from computer-aided design (CAD) input data. Thus, component shapes can be defined by calculations that represent the theoretical ideal for a given application. These techniques have been widely used for prototypes but also used to make production-quality and cost-effective components in metal [16] and in plastic [17].

The additive nature of LBMTs allows the creation of objects with complicated internal features that may not be viable by other means. If these components are required in small batches, layer-based approaches often offer the optimum manufacturing process. Furthermore, design iterations do not require modification to tooling or the manufacturing process and so are easily accommodated. This is a valuable feature of LBMTs when producing prototypes for experimentation.

Several commercially available LBMTs exist. These can be classified into several groups depending upon the mode of fusing energy provision (laser, non-laser radiation, heated nozzle) and also by material feedstock (metallic/polymeric powder, polymer resin, plaster/ ink) and are discussed more exhaustively elsewhere [18]. In this study, digital light processing (DLP) was employed, which uses a dynamic masking technique to selectively cure a photosensitive polymer resin, in this case polymethylmethacrylate (PMMA).

A computer model of the part to be manufactured is generated in a CAD package and then subjected to a 'slicing' routine. Each of these slices is used to generate a two color bitmap file. The contrast in the generated 
Table 1. Typical surface roughness of plane surfaces manufactured by DLP, where $R_{a}=$ arithmetic average, $R_{q}=$ root mean squared, $R_{z}=$ mean peak to valley, and $R_{t}=$ maximum peak to valley

\begin{tabular}{lcc}
\hline Specimen type & Measurement & Value \\
\hline \hline & $\mathrm{R}_{\mathrm{a}}$ & $577 \mathrm{~nm}$ \\
$50 \mu \mathrm{m}$ (ERM on) & $\mathrm{R}_{\mathrm{a}}$ & $703 \mathrm{~nm}$ \\
& $\mathrm{R}_{\mathrm{z}}$ & $3.35 \mu \mathrm{m}$ \\
& $\mathrm{R}_{\mathrm{t}}$ & $3.48 \mu \mathrm{m}$ \\
$50 \mu \mathrm{m}$ (ERM off) & $\mathrm{R}_{\mathrm{a}}$ & $716 \mathrm{~nm}$ \\
& $\mathrm{R}_{\mathrm{q}}$ & $892 \mathrm{~nm}$ \\
& $\mathrm{R}_{\mathrm{z}}$ & $6.16 \mu \mathrm{m}$ \\
& $\mathrm{R}_{\mathrm{t}}$ & $7.23 \mu \mathrm{m}$ \\
$25 \mu \mathrm{m}$ (ERM on) & $\mathrm{R}_{\mathrm{a}}$ & $434 \mathrm{~nm}$ \\
& $\mathrm{R}_{\mathrm{a}}$ & $541 \mathrm{~nm}$ \\
& $\mathrm{R}_{\mathrm{z}}$ & $4.2 \mu \mathrm{m}$ \\
$25 \mu \mathrm{m}$ (ERM off) & $\mathrm{R}_{\mathrm{t}}$ & $4.4 \mu \mathrm{m}$ \\
& $\mathrm{R}_{\mathrm{a}}$ & $749 \mathrm{~nm}$ \\
& $\mathrm{R}_{\mathrm{a}}$ & $895 \mathrm{~nm}$ \\
& $\mathrm{R}_{\mathrm{z}}$ & $3.97 \mu \mathrm{m}$ \\
& $\mathrm{R}_{\mathrm{t}}$ & $4.18 \mu \mathrm{m}$ \\
\hline
\end{tabular}

bitmap image generated is used to actuate a digital mirror device (DMD chip), which can be exploited to cause selective exposure of a monomer resin feedstock ensuring localized polymerization. This process is repeated in each slice of the component and a solid part is formed.

DLP has been shown to be a suitable process for the production of QMS mass filters [19], and it is proposed that it is also suitable for ion trap manufacture. DLP components retain good geometric tolerances upon assembly and smooth surfaces, both of which are necessary for ion trap electrode structures. The surface roughness of components produced in this way is anisotropic. That is to say, surface roughness and other material properties such as tensile strength are dependent upon the geometry orientation in the build envelope. The apparatus used in this study is capable of a layer thickness of $25 \mu \mathrm{m}$ and is also equipped with so-called enhanced resolution mode (ERM), which further improves surface quality. The required geometries for this design were of uniform cross section and thus they are subject to surface roughness perpendicular to the build direction. Typical surface roughness values for this orientation are shown in Table 1.

The electrode surfaces are rendered conducting via the deposition of a layer of gold by thermal evaporation. The density of PMMA is $\approx 1.2 \mathrm{~g} / \mathrm{cm}^{3}$, whereas the density of stainless steel is $\approx 7.8 \mathrm{~g} / \mathrm{cm}^{3}$. Therefore, structures manufactured in this way are lightweight compared with their conventional metal counterparts. This is an important consideration for weight-critical applications such as aerospace.

Fico et al. [20] used an alternative RM technique, stereo lithographic apparatus (SLA), for the manufacture of rectilinear ion traps (RITs). The SLA process involves rastering a laser over a resin bath causing selective polymerization. The feature sizes of parts manufactured by this method are limited by the mini- mum laser spot size obtainable and minimum cure dimensions. Therefore, SLA components usually exhibit a higher surface roughness and lower tolerance compared with similar components produced by DLP. Sager and Rosen report surface roughness values (Ra) for a fully optimized SLA to be of the order of $1 \mu \mathrm{m}$ [21]. This arises due to the progression of the laser across the surface in tracks, which will be overlapped. SLA tracks are significantly larger $(\approx 200 \mu \mathrm{m}$ wide $)$ than the volumetric pixels (voxels $\approx 15 \mu \mathrm{m} \times 15 \mu \mathrm{m} \times 25 \mu \mathrm{m}$ ) used in DLP. Furthermore, the previous work did not exploit the design freedoms granted by layer-based techniques to produce a hyperbolic profile. It is well known that hyperbolic profile electrodes offer significant advantages over other electrode geometries (e.g., cylindrical) due to the better representation of the ideal quadrupolar electric field. For a QMS, this has been shown experimentally [22] and theoretically by quantifying both improvements to resolution and transmission [23].

In this paper, the first use of DLP for the fabrication of a linear ion trap mass analyzer is reported. The as-fabricated DLP trap was tested with a miniature mass spectrometer equipped with a discontinuous atmospheric-pressure interface (DAPI) [24] typical of a sample acquisition module and described in more detail later. The resolution, mass range, and tandem capability of the ion trap were characterized for a range of analytes in the mass range to $m / z$ 1900. The design process used here is presented as a model for ion trap mass spectrometer prototyping.

\section{Experimental}

\section{Design and Fabrication}

A detailed CAD design was undertaken to define dimensions for every component. Figure 1 shows the assembly, and also the design for the individual components, including electrodes and electrode housing for the DLP linear ion trap (LIT) with hyperbolic electrodes. Figure $1 \mathrm{~b}$ shows an exploded assembly of the design highlighting crucial advantages of using DLP for LIT manufacture, namely the ability to directly manufacture screw threads and fine features such as the ion access slits $(25.5 \mathrm{~mm} \times 188 \mu \mathrm{m})$. The flexibility of this approach facilitates the interchange of components and reduces experiment down time. The prototype electrodes of the ion trap used in this study are shown in Figure 2.

The DLP LIT has distance between $x$ electrodes of 6 $\mathrm{mm}$ and $5.052 \mathrm{~mm}$ between $y$ electrodes. This electrode spacing was selected by following the same ratio utilized by Schwartz et al. [25] by increasing $r_{0 x}$ value for the slotted hyperbolic LIT electrodes by a factor of 1.1875. The benefits of electrode stretching are well known and discussed by March and Todd for a conventional quadrupole ion trap [26]. In the case of LITs, the stretch helps to compensate for loss of linearity for higher order electric fields caused by the slits in the $x$ 
(a)

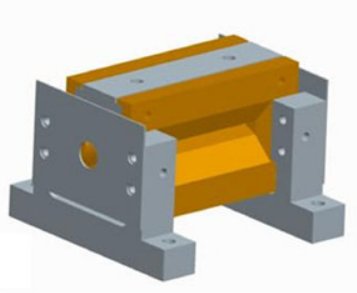

(b)

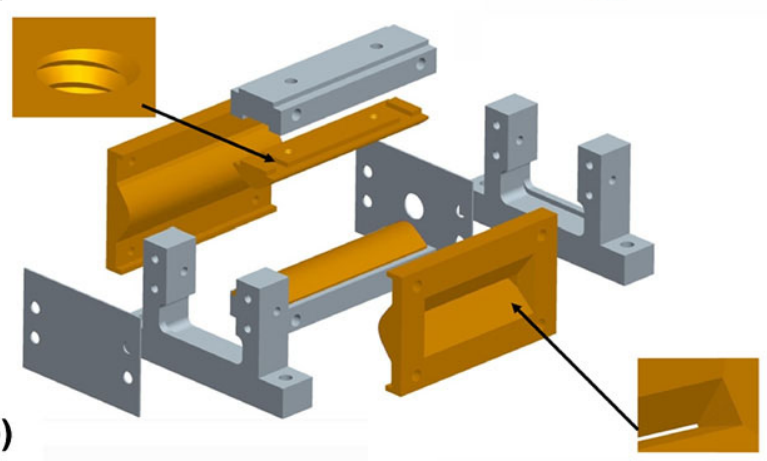

(c)
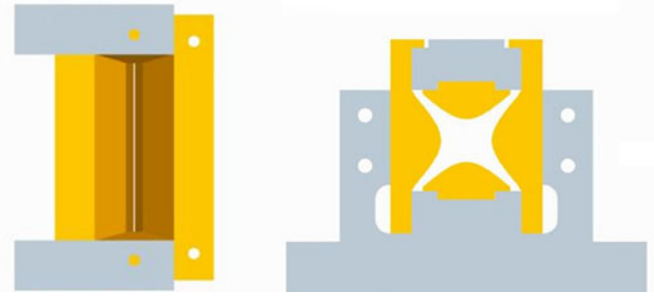

Figure 1. (a) CAD model and actual prototype of the DLP LIT, (b) exploded assembly of the DLP LIT showing threaded hole and ion entrance/extract details, (c) the DLP LIT electrode profile.

electrodes. This is explained in Figure 3, which shows electric field variations in $x$ direction from the center of the DLP LIT to the electrode edge for original trap without slits, original trap with slits, and stretched trap with slits. It can be seen from the plots that field variation is linear with no slit involved and becomes nonlinear near the electrode edge when slit is included. The effect of stretching the electrode to compensate for

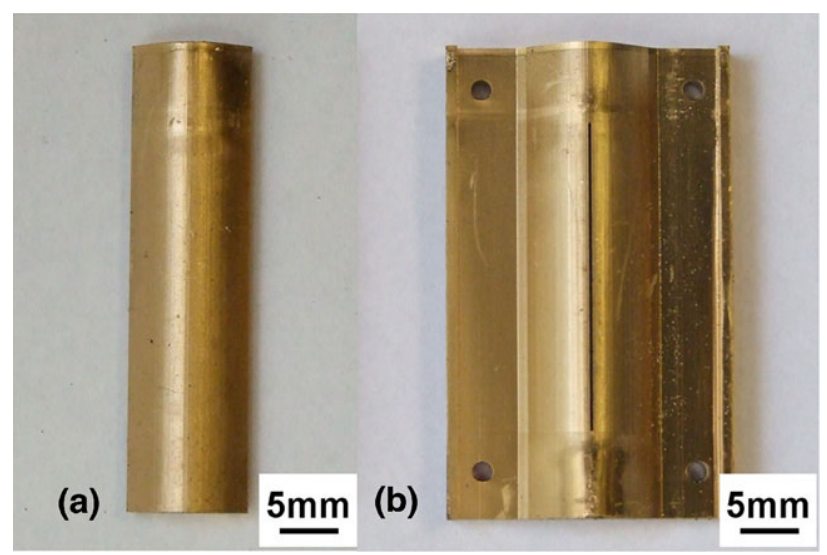

Figure 2. Photographs of the DLP LIT components showing gold coated individual electrodes (a) $y$ electrode, (b) $x$ electrode.

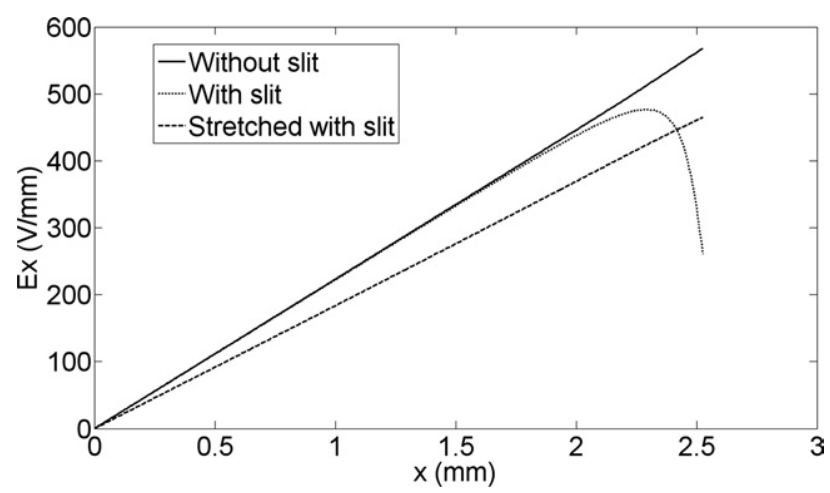

Figure 3. Electric field variations in $x$ direction from the center of the DLP LIT to the electrode edge with $716 \mathrm{~V}_{p-p}$ rf amplitude. Variations were done with original $r_{0 x}=2.526 \mathrm{~mm}$ for $x$ electrode without slit, with slit and stretched with slit.

the slit is clearly shown by the improved field linearity in the $x$ direction; in this case, however, with a lower slope, which can be adjusted by applying higher voltages to the electrodes. The effect of stretching the electrodes also affects ion secular motion. This is illustrated in Table 2, showing a comparison of the ion secular frequencies calculated for the rhodamine $B$ ion with $m / z=443$ oscillating in the DLP LIT driven at 1185 $\mathrm{kHz}$ with $717 \mathrm{~V}_{p-p}$ rf and $15 \mathrm{~V}$ dc on the endcap electrodes. The secular frequencies were determined from the fast Fourier transform of the ion trajectories following the method of Brkić et al. [27]. The effect of the slotted electrodes and of stretching the trap is clearly seen in the frequency shift, with a major decrease occurring in radial secular frequencies when stretching the trap. This is because radial secular frequencies in ion traps are inversely proportional to the square value of $r_{0}$ [28], and axial secular frequencies in linear traps are directly proportional to the square root of the 'geometric factor' of the trap [29], which is affected by every change in electrode shape and position.

The details of the fabrication procedure have been reported previously [19]. For our DLP LIT, a commercial Envisiontec Perfactory system (Gladbeck, Germany and Ferndale, MI, USA) was used to fabricate electrodes using PMMA. Gold coating for the PMMA electrodes was done by thermal evaporation with an Edwards E306A Coating System (Crawley, UK and Tewksbury, MA, USA). The thickness of the gold coating is $\sim 0.1$

Table 2. Numerical results for radial $(x, y)$ and axial $(z)$ secular frequencies for rhodamine B $(m / z=443)$ ions within DLP LIT with changing configuration for $x$ electrodes

\begin{tabular}{|c|c|c|c|c|}
\hline \multirow[t]{2}{*}{$\mathrm{x}$-Electrodes } & \multicolumn{2}{|c|}{$\begin{array}{c}\text { Electrode } \\
\text { spacing }(\mathrm{mm})\end{array}$} & \multicolumn{2}{|c|}{$\begin{array}{c}\text { Secular } \\
\text { frequencies } \\
(\mathrm{kHz})\end{array}$} \\
\hline & $r_{O x}$ & $r_{O y}$ & $\omega_{x, y} / 2 \pi$ & $\omega_{z} / 2 \pi$ \\
\hline Without slits & 2.526 & 2.526 & 495 & 0.79 \\
\hline Including slits & 2.526 & 2.526 & 491 & 0.78 \\
\hline Stretched including slits & 3 & 2.526 & 348 & 0.70 \\
\hline
\end{tabular}


$\mu \mathrm{m}$, and the resistance from end to along the electrode surfaces after assembly and connection into the vacuum system was found to be negligible $(<0.1 \Omega)$.

A Mini 10 mass spectrometer equipped with a discontinuous atmospheric pressure interface (DAPI) was used to test the ion trap. The detailed configuration of the mass analyzer assembly can be found in previous reports. Ions generated at atmospheric pressure are transferred into the ion trap driven by a differential rf signal through the DAPI interface, and an electron multiplier is used to detect ions ejected from the trap. The DAPI system consists of two stainless steel capillaries connected to each other via a conductive silicone tube. The conductive silicon tube goes through a normally closed pinch valve, which controls the open/ closed status of the DAPI. During operation, a $24 \mathrm{~V}$ dc pulse signal lasting for a few $\mathrm{ms}$ is applied to the pinch valve at the beginning of each scan cycle to open the DAPI for ion introduction; the interface then closes for the remainder of the scan cycle. After the pumping system is restored, the manifold pressure drops in the course of a sufficiently long cooling period, the multiplier is turned on, and both the rf signal amplitude and resonance ac signal amplitude are scanned to record the mass spectrum. A capacitor divider built with two capacitors connected in series is used to measure the output voltage of the rf system. The capacitance of two capacitors is 5.58 and $1330 \mathrm{pF}$ respectively, which gives a ratio of 1:239 between the measured voltage and the real rf voltage. The dc voltages on both ion trap end electrodes are constant at $+15 \mathrm{~V}$ during the whole scan cycle. A home-built electrospray source using fusedsilica capillaries and SwageLok tee was used to ionize samples prepared using 1:1 methanol water solution with $1 \%$ of acetic acid. Samples were sprayed at a flow rate of $1 \mu \mathrm{L} / \mathrm{min}$ controlled by a syringe pump with a bias voltage of $+3 \mathrm{kV}$ and a nitrogen sheath gas pressure of 80 psi applied.

\section{Results and Discussion}

\section{MS and Tandem MS Capabilities}

A 10 ppm rhodamine B solution ionized by ESI was analyzed with the DLP ion trap first. The $\mathrm{rf}$ frequency was tuned at $1185 \mathrm{kHz}$ when the DLP ion trap was used for mass analysis. During the mass analysis, the pinch valve was opened $8 \mathrm{~ms}$ for ion introduction, and then the rf voltage was scanned from 338 to $1185 \mathrm{~V}_{p-p}$ in 80 ms after a $1.2 \mathrm{~s}$ cooling period. A $470 \mathrm{kHz}$ resonance ac signal was applied during rf scanning to establish resonance ejection, corresponding to a $q$ value of 0.85 . The ac signal amplitude was also scanned from 1 to 5 $\mathrm{V}_{p-p}$ to achieve optimal resolution over the whole mass range. The mass spectrum obtained is shown in Figure $4 \mathrm{a}$. The rf voltage corresponding to $m / z 443$ is $717 \mathrm{~V}_{p-p}$. The tandem MS capability of the ion trap was also demonstrated. The parent ion $m / z 443$ was isolated and then fragmented by applying SWIFT (stored waveform inversion Fourier transform) waveforms followed by an excitation ac signal, respectively. A SWIFT waveform, with amplitude $5.0 \mathrm{~V}$ and a frequency notch between 150 and $155 \mathrm{kHz}$ was first applied during ion isolation, the rf amplitude was adjusted to set the secular frequency of the precursor ions within the frequency notch, and the SWIFT signal was applied for $5.0 \mathrm{~ms}$ to eject all other ions from the trap. The isolation spectrum is shown in Figure $4 \mathrm{~b}$. The excitation ac signal with amplitude of $0.3 \mathrm{~V}_{p-p}$ and frequency $153 \mathrm{kHz}$ was applied for $20.0 \mathrm{~ms}$ to excite the parent ions of $\mathrm{m} / \mathrm{z} 443$ to induce fragmentation through collisions with background air molecules. Fragment ions with mass to charge ratios of 398 and 412 can be observed from the fragmentation spectrum shown in Figure 4c. A zoom in view of the peak at $m / z 443$ is shown as an insert in Figure $4 \mathrm{a}$ and $\mathrm{b}$. The zoom in view from $\mathrm{m} / \mathrm{z} 390$ to 450 is shown in Figure 4c. In Figure 4a and b, the FWHM is about 1.8 for the precursor ion peak at $m / z 443$, which corresponds to a resolution of 250 . In Figure $4 c$, the FWHM is about 1.5 for the product ion peak at $m / z 398$, which corresponds to a resolution of 260 . The peak splitting at $m / z 442$ may be due to the fragments with different $\mathrm{m} / \mathrm{z}$ that cannot be well separated at this resolution.

The Mini 10 system including the DAPI inlet was subsequently fitted with its standard RIT (same length and external dimensions as the DLP trap) and the system was used to analyze the same analyte sample for comparison. With the RIT as the mass analyzer, an rf signal with frequency of $1340 \mathrm{kHz}$, and the resonance ac signal with frequency of $480 \mathrm{kHz}$ (corresponding to a $q$ value of 0.8 ) were applied. The mass spectrum obtained is shown in Figure $4 \mathrm{~d}$. The rf voltage corresponding to the $m / z=443$ is $2280 \mathrm{~V}_{p-p}$ in the RIT experiment. An isolation spectrum (Figure 4e) and fragmentation spectrum of rhodamine B (Figure 4f) were also obtained, and almost identical fragmentation peaks were obtained as for DLP LIT. Spectra were also obtained to measure the resolution, which in the RIT case was superior, and this is probably due to the lower tolerances of manufacture in the case of the RIT. Using the DLP trap, experimental mass spectrum was also obtained for 5 ppm mixture of methamphetamine $(\mathrm{m} / \mathrm{z}$ $150)$ and cocaine $(m / z=182,304)$ as shown in Figure 5. Peaks were stable and reproducible in all cases. Protonated methamphetamine and cocaine are observed at $\mathrm{m} / \mathrm{z}$ 150 and 304. The peak at $m / z 182$ corresponds to a fragment of cocaine.

A higher mass range can be achieved by reducing the size of an ion trap and/or by increasing the operating voltage. In the case of the DLP ion trap, rf voltage excitation corresponding to a mass range up to $\mathrm{m} / \mathrm{z} 1900$ was demonstrated with the Mini 10 test system. Ultramark 1621 is commonly used as an instrument calibrant in the $1500-1700 \mathrm{~m} / \mathrm{z}$ mass range [30]. A solution of peptide MRFA and Ultramark was prepared and was subject to electrospray ionization ESI before injection into the DLP LIT. Protonated MRFA was not observed 

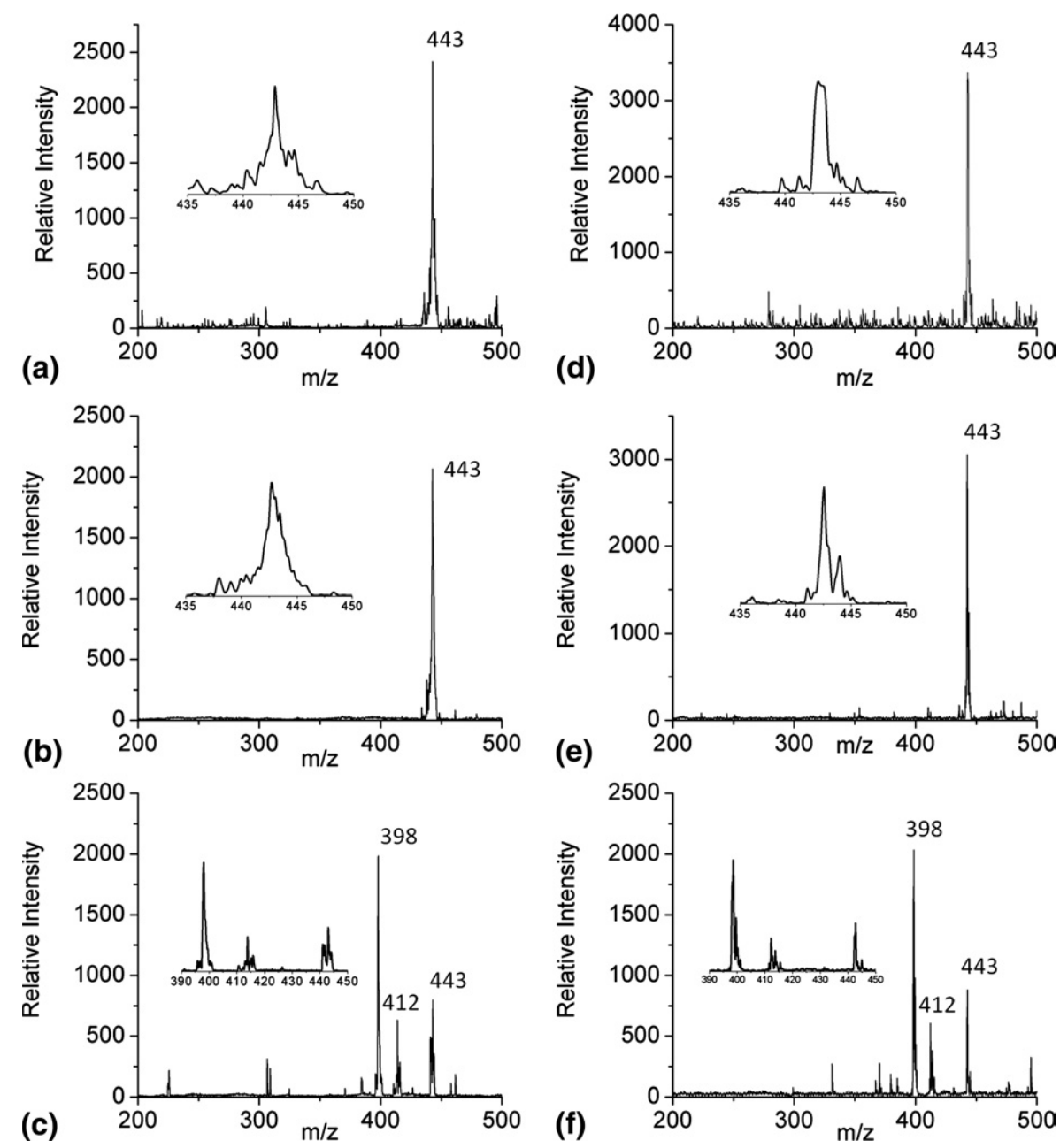

Figure 4. Mass spectra of rhodamine B collected using the DLP LIT (a), (b), (c) and spectra obtained using a conventional RIT operating under identical conditions (d), (e), (f).

in the spectrum and the $\mathrm{S} / \mathrm{N}$ ratio of the Ultramark peaks was low, which may be due to a reduced trapping capacity. The spectrum obtained is shown in Figure 6 .

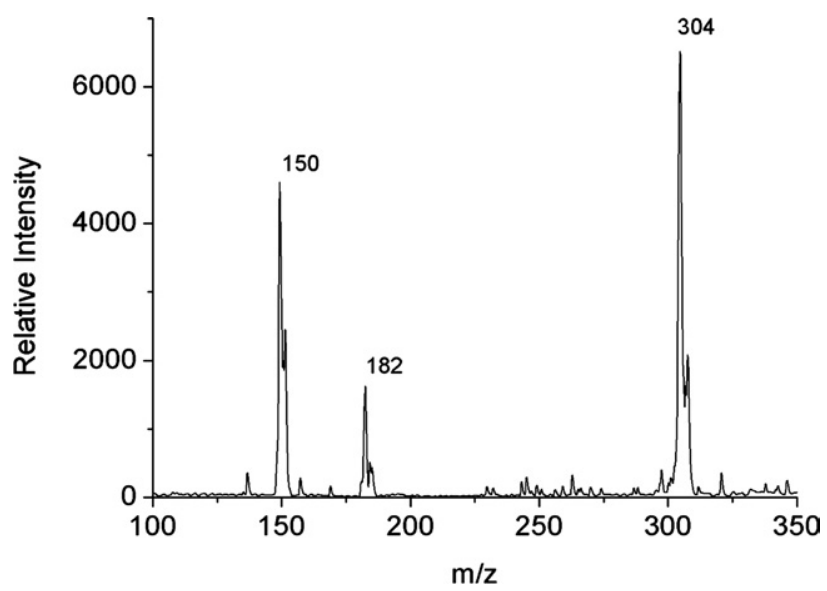

Figure 5. Mass spectrum for $5 \mathrm{ppm}$ mixture of cocaine and methamphetamine.

\section{Conclusions}

A linear ion trap with gold coated hyperbolic form electrodes has been fabricated using the DLP technique and shown to work in the mass range to $\mathrm{m} / \mathrm{z} 500$ (rhodamine B) with a maximum measured resolution (FWHM) of 260. The results were compared with those obtained from a RIT tested under identical conditions (same external dimensions but DLP has smaller internal dimensions). The resolution in the case of the RIT was greater, but the operating voltage in the case of the DLP was much lower for a given mass: $716 \mathrm{~V}_{p-p} \mathrm{rf}$ for $m / z=$ 443 compared with $2382 \mathrm{~V}_{p-p} \mathrm{rf}$ in the case of the RIT. This is a result of the hyperbolic form of the electrodes providing an effectively lower $r_{0}$ for a given overall trap volume. Lower $r_{0}$ gives a lower ion capacity, but this is compensated for by higher trapping efficiency enabled by the improved field linearity due to the hyperbolic electrodes.

The use of DLP LIT can be suitable in field (harsh environment) applications where a high mass range is required, and work is ongoing to address this issue. The 


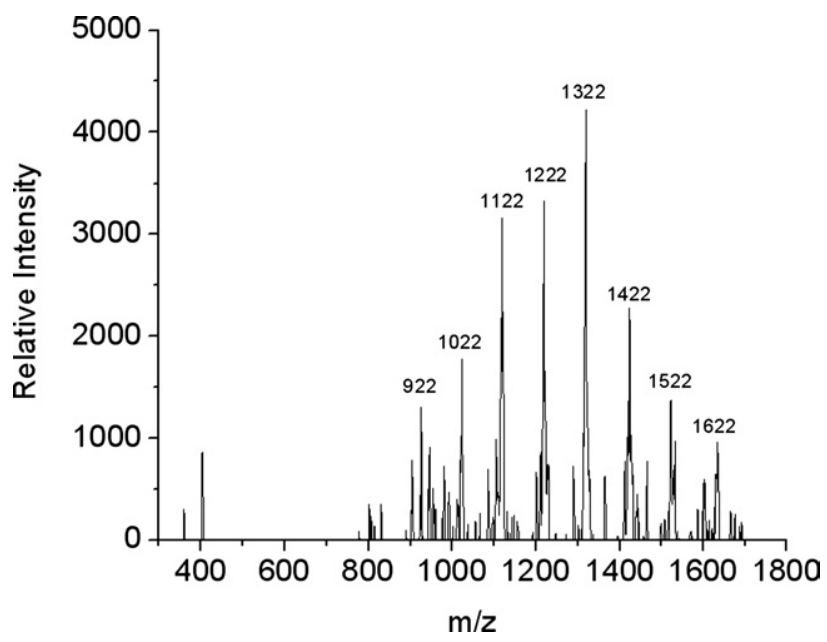

Figure 6. Mass spectrum of Ultramark obtained from Mini 10 and the DLP LIT. The drive parameters include rf frequency of 990 $\mathrm{KHz}$, resonance ac frequency of $360 \mathrm{KHz}$, rf feedback of $25.7 \mathrm{~V}$ and rf amplitude of $\sim 2670 \mathrm{~V}_{p-p}$.

DLP technique has been found to be particularly suitable for realization of mass analyzers with hyperbolic or other noncircular geometry electrodes, and is capable of accurate feature sizes in the sub-mm range. Rapid and low costs of manufacture with good tolerances and surface finish, minimal secondary processing, and quick component inter-assembly are all advantages of DLP. The relative early stage of development of DLP as a manufacturing technique means that its full potential is not yet realized and that further work remains to exploit this method in miniature and portable mass spectrometry.

\section{Acknowledgments}

The authors gratefully acknowledge technical consultations with S. Smith and R. G. Cooks of Purdue University. The UK Engineering and Physical Science Research Council (EPSRC) is acknowledged for the provision of funding. The Head of Department, Electrical Engineering and Electronics at the University of Liverpool (EEE UoL) Professor S. Hall, is acknowledged for the offer of sabbatical leave for one of the investigators (ST). The authors gratefully acknowledge the help of colleagues in the Aston Labs (Purdue University) and other members of the Mass Spectrometry research group at Liverpool.

\section{References}

1. Eckenrode, B. A. Environmental and Forensic Applications of FieldPortable GC-MS: An Overview. J. Am. Soc. Mass Spectrom. 2001, 12, 683-693.

2. Blain, M. G.; Riter, L. S.; Cruz, D.; Austin, D. E.; Wu, G.; Plass, W. R.; Cooks, R. G. Towards the Hand-Held Mass Spectrometer: Design Considerations, Simulation, and Fabrication of Micrometer-Scaled Cylindrical Ion Traps. Int. J. Mass Spectrom. 2004, 236, 91-104.
3. Ferran, R. J.; Boumsellek, S. High-Pressure Effects in Miniature Arrays of Quadrupole Analyzers for Residual Gas Analysis from $10^{-9}$ to $10^{-2}$ Torr. J. Vac. Sci. Technol. A 1996, 14, 1258-1265.

4. Taylor, S.; Tindall, R. F.; Syms, R. R. A. Silicon-Based Quadrupole Mass Spectrometry Using Microelectromechanical Systems. J. Vac. Sci. Technol. B 2001, 19, 557-562.

5. Geear, M.; Syms, R. R. A.; Wright, S.; Holmes, A. S. Monolithic MEMS Quadrupole Mass Spectrometers by Deep Silicon Etching. IEEE/ASME J. Microelectromech. Syst. 2005, 14, 1156-1166.

6. Yoon, H. J.; Kim, J. H.; Choi, E. S.; Yang, S. S.; Jung, K. W. Fabrication of a Novel Micro Time-of-Flight Mass Spectrometer. Sensors Actuators A 2002, 97/98, 441-447.

7. Wapelhorst, E.; Hauschild, J. P.; Müller, J. Complex MEMS: A Fully Integrated TOF Micro Mass Spectrometer. Sensors Actuators A 2007, 138, 22-27.

8. Hauschild, J. P.; Wapelhorst, E.; Müller, J. The Novel Synchronous Ion Shield Mass Analyzer. I. Mass Spectrom. 2009, 44, 1330-1337.

9. Pau, S.; Pai, C. S.; Low, Y. L.; Moxom, J.; Reilly, P. T. A.; Whitten, W. B.; Ramsey, J. M. Microfabricated Quadrupole Ion Trap for Mass Spectrometer Applications. Phys. Rev. Lett. 2006, 96, 120801.

10. Lammert, S. A.; Rockwood, A. A.; Wang, M.; Lee, M. L.; Lee, E. D. Tolley, S. E.; Oliphant, J. R.; Jones, J. L.; Waite, R. W. Miniature Toroidal Radio Frequency Ion Trap Mass Analyzer. J. Am. Soc. Mass Spectrom. 2006, 17, 916-922.

11. Cruz, D.; Chang, J. P.; Fico, M.; Guymon, A. J.; Austin, D. E.; Blain, M. G. Design Microfabrication, and Analysis of Micrometer-Sized Cylindrical Ion Trap Arrays. Rev. Sci. Instrum. 2007, 78, 015107.

12. Austin, D. E.; Peng, Y.; Hansen, B. J.; Miller, I. W.; Rockwood, A. L.; Hawkins, A. R.; Tolley, S. E. Novel Ion Traps Using Planar Resistive Electrodes: Implications for Miniaturized Mass Analyzers. J. Am. Soc. Mass Spectrom. 2008, 19, 1435-1441.

13. Badman, E. R.; Johnson, R. C.; Plass, W. R.; Cooks, R. G. A Miniature Cylindrical Quadrupole Ion Trap: Simulation and Experiment. Anal. Chem. 1998, 70, 4896-4901.

14. Van Amerom, F. H. W.; Chaudhary, A.; Cardenas, M.; Bumgarner, J. Short, R. T. Microfabrication of Cylindrical Ion Trap Mass Spectrometer Arrays for Handheld Chemical Analyzers. Chem. Eng. Comm. 2008, 195, 98-114.

15. Ouyang, Z.; Gao, L.; Fico, M.; Chappell, W. J.; Noll, R. J.; Cooks, R. G. Quadrupole Ion Traps and Trap Arrays: Geometry, Material, Scale, Performance. Eur. J. Mass Spectrom. 2007, 13, 13-18.

16. Wong, M.; Tsopanos, S.; Sutcliffe, C. J.; Owen, E. Selective Laser Melting of Heat Transfer Devices. Rapid Prototyping J. 2007, 13, 291-297.

17. Karania, R.; Kazmer, D. Low Volume Plastics Manufacturing Techniques. J. Mech. Des. 2007, 129, 1225-1233.

18. Grimm, T. User's Guide to Rapid Prototyping. Society of Manufacturing Engineers: Dearborn, 2004; p. 52.

19. Brkić, B.; France, N.; Clare, A. T.; Sutcliffe, C. J.; Chalker P. R.; Taylor, S. Development of Quadrupole Mass Spectrometers Using Rapid Prototyping Technology. J. Am. Soc. Mass Spectrom. 2009, 20, 1359-1365.

20. Fico, M.; Yu, M.; Ouyang, Z.; Cooks, R. G.; Chappell, W. J. Miniaturization and Geometry Optimization of a Polymer-Based Rectilinear Ion Trap. Anal. Chem. 2007, 79, 8076-8082.

21. Sager, B.; Rosen, D. W. Use of Parameter Estimation for Stereolithography Surface Finish Improvement. Rapid Prototyping J. 2008, 14, 213-220.

22. Brubaker, W. M.; Chamberlin, W. S. Theoretical and Experimental Comparisons of Quadrupole Mass Analyzers with Round and Hyperbolic Field-Forming Surfaces. NASA Report 1970.

23. Gibson, J. R.; Taylor, S. Prediction of Quadrupole Mass Filter Performance for Hyperbolic and Circular Cross Section Electrodes. Rapid Commun. Mass Spectrom. 2000, 14, 1669-1673.

24. Griffiths, J. A Mass Spectrometer in Every Hand. Anal. Chem. 2008, 80, 7904.

25. Schwartz, J. C.; Senko, M. W.; Syka, J. E. P. A Two-Dimensional Quadrupole Ion Trap Mass Spectrometer. J. Am. Soc. Mass Spectrom. 2002, 13, 659-669.

26. March, R. E.; Todd, J. F. Quadrupole Ion Trap Mass Spectrometry, 2nd ed. Wiley-Interscience: New York, 2005; p. 108.

27. Brkić, B.; Taylor, S.; Ralph, J. F.; France, N. High-Fidelity Simulations of Ion Trajectories in Miniature Ion Traps Using the Boundary-Element Method. Phys. Rev. A 2006, 73, 012326.

28. March, R. E. An Introduction to Quadrupole Ion Trap Mass Spectrometry. J. Mass Spectrom. 1997, 32, 351-369.

29. Berkeland, D. J.; Miller, J. D.; Bergquist, J. C.; Itano, W. M.; Wineland, D. J. Minimization of Ion Micromotion in a Paul Trap. J. Appl. Phys. 1998, 83, 5025-5033.

30. Moini, M. Ultramark 1621 as a Calibration/Reference Compound for Mass Spectrometry. II. Positive- and Negative-Ion Electrospray Ionization. Rapid Commun. Mass Spectrom. 1994, 8, 711-714. 